\title{
Effect of physical activity promotion on adiponectin, leptin and other inflammatory markers in prediabetes: a systematic review and meta-analysis of randomized controlled trials
}

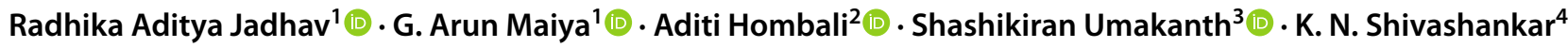

Received: 1 August 2020 / Accepted: 21 October 2020 / Published online: 19 November 2020

(c) The Author(s) 2020

\begin{abstract}
Aims Inflammatory stage in prediabetes is associated with increase in level of adipokines and pro-inflammatory cytokines. Physical activity promotion considered as a first-line therapeutic strategy to treat prediabetes. We have conducted the systematic review and meta-analysis to strengthen the evidence on the impact of physical activity promotion on inflammatory markers in prediabetes.

Methods Studies were identified using electronic search and manual search techniques by choosing keywords for prediabetes, physical activity and inflammatory marker. Randomized controlled trials on individuals diagnosed with prediabetes and provided intervention in the form of physical activity were included in this review. Adiponectin, leptin, C-reactive protein, interleukin- 6 and tumour necrosis factor- $\alpha$ were the considered outcome measures.

Results Our search retrieved 1,688 citations, 31 full-text articles assessed for eligibility of inclusion. Nine studies satisfied the pre-specified criteria for inclusion. Meta-analysis found that physical activity with or without dietary or lifestyle modification reduces level of leptin (MD-2.11 ng/mL, 95\% CI -3.81--0.42) and interleukin-6 (MD -0.15 pg/mL, 95\% CI -0.25--0.04). It has no effect on level of adiponectin (MD $0.26 \mu \mathrm{g} / \mathrm{mL}, 95 \% \mathrm{CI}-0.42-0.93$ ), C-reactive protein (MD $-0.05 \mathrm{mg} / \mathrm{L}, 95 \% \mathrm{CI}-0.33-0.23$ ) and tumour necrosis factor- $\alpha$ (MD $0.67 \mathrm{pg} / \mathrm{mL}, 95 \% \mathrm{CI}-2.56-3.89$ ).

Conclusions This review suggests that physical activity promotion with dietary and lifestyle modification may reduce the level of leptin and interleukin- 6 but are uncertain if there is any effect on levels of adiponectin, C-reactive protein and tumour necrosis factor- $\alpha$ in the individuals with prediabetes.
\end{abstract}

Keywords Diabetes $\cdot$ Lifestyle $\cdot$ Inflammation $\cdot$ Cytokines $\cdot$ Adipokines

Managed by Antonio Secchi.

Electronic supplementary material The online version of this article (https://doi.org/10.1007/s00592-020-01626-1) contains supplementary material, which is available to authorized users.

G. Arun Maiya

arun.maiya.g@gmail.com; arun.maiya@manipal.edu

1 Department of Physiotherapy, Centre for Diabetic Foot Care and Research, Manipal College of Health Professions, Manipal Academy of Higher Education, Manipal 576104, Karnataka, India

2 Independent Systematic Reviewer, Luton, Bedfordshire, UK

3 Department of Medicine, Melaka Manipal Medical College, Manipal Academy of Higher Education, Manipal 576104, Karnataka, India

4 Department of Medicine, Kasturba Medical College, Manipal Academy of Higher Education, Manipal 576104, Karnataka, India

\author{
Abbreviations \\ T2DM Type 2 diabetes mellitus \\ ADA American Diabetes Association \\ WHO World Health Organization \\ IL-6 Interleukin 6 \\ CRP C-reactive protein \\ TNF- $\alpha$ Tumour necrosis factor- $\alpha$ \\ MCID Minimally clinical important difference \\ PA Physical activity
}

\section{Introduction}

Prediabetes is an intermediate stage of abnormal glucose homeostasis, where blood glucose level is more than a normal range and lesser than the range to confirm type 2 diabetes mellitus (T2DM) [1]. World Health Organization (WHO) and the American Diabetes Association (ADA) have given 
criteria to define prediabetes $[2,3]$. Prediabetes has a high chance of converting into T2DM [4].

Obesity in prediabetes is associated with low-grade chronic systemic inflammation. The level of pro-inflammatory markers increases in prediabetes [5]. Pro-inflammatory state in prediabetes is predominantly because of the increase in insulin resistance [6]. It is considered due to changes in circulating factors released from adipose tissue [7]. Secretions from adipose tissue consist of adipokines and cytokines like C-reactive proteins (CRP), interleukin-6 (IL$6)$, adiponectin, leptin and tumour necrosis factor- $\alpha$ (TNF- $\alpha$ ) [8-10]. Adiponectin and leptin are the adipokines; along with their metabolic property, they are also responsible for inflammation and oxidative stress [11]. High level of leptin activates macrophages and monocytes to produce IL- 6 and TNF- $\alpha$ [12]. CRP is an inflammatory protein increase with an increase in inflammation. Thus, like T2DM, prediabetic individuals are also at high risk for development of cardiovascular complications [13].

Physical inactivity is one of the important modifiable risk factors to reduce the burden of prediabetes or type 2 diabetes mellitus (T2DM). Previous literature has reported the impact of physical activity on the risk of T2DM [14, 15]. Physical activity promotion is a first-line therapeutic strategy, which stands before the pharmacological intervention to treat prediabetes $[15,16]$.

Previous reviews have focused on examining the impact of physical activity intervention on glycaemic parameters and the resulting reduction in aggravation of prediabetes to T2DM $[14,17]$. Impact of lifestyle intervention on the level of different inflammatory markers has documented in long-term clinical trials [18-20]. However, there is a diversity in physical activity intervention used and inflammatory markers on which their effects have been seen. Therefore, we have conducted a systematic review and meta-analysis to strengthen the evidence on the impact of physical activity promotion on inflammatory markers in individuals with prediabetes. The main objective of this review is to assess the effect of physical activity promotion on inflammatory markers in individuals with prediabetes.

\section{Methods}

This review is conducted and reported as per the Preferred Reporting Items for Systematic Reviews and Meta-Analysis (PRISMA) checklist [21].

\section{Information sources}

Studies were identified using electronic search as well as manual search techniques. The following databases were searched-PubMed (Medline), Scopus, Web of Science,
Cumulative Index to Nursing and Allied Health Literature (CINHAL), Cochrane Central Register of Controlled Trials (CENTRAL) and Embase in December 2019.

\section{Search strategy}

Comprehensive searches were carried out by choosing keywords and subject headings for prediabetes, physical activity and inflammatory marker. Variations in the search terms were identified using truncations and wildcard symbols. The search terms were then combined using Boolean operators. Search filters were used to exclude animal studies and studies published in languages other than English. The identified search terms and the details of the search strategies used in databases have presented in online resource 1 . We manually reviewed the references lists of the included studies to identify additional eligible primary studies.

\section{Study selection}

The citations were imported from all the databases to Ryan software [22], and the duplicate citations were identified and removed. The screening process was undertaken in two stages titles: abstract screening and full-text screening. The selection was based on pre-specified review's inclusion and exclusion criteria. We identified multiple reports from the same study and reported them in online resource 2 .

\section{Eligibility criteria}

\section{Population}

Individuals of both gender and age above 20 years, diagnosed with prediabetes by either WHO or ADA criteria, were included. Studies that included individuals diagnosed with type two diabetes mellitus or with normal glucose tolerance were excluded from this review.

\section{Intervention}

We included studies with intervention in the form of physical activity promotion. Physical activity promotion included home-based physical activity or supervised activity session. Any single or multiple forms of physical activity administered in the form of supervised exercises, scheduled activity, weight loss intervention were included. Personal counselling and advice to encourage participation in physical activities along with dietary advice and lifestyle modification were also included. Studies that included other pharmacological intervention along with physical activity were excluded. 


\section{Comparison}

Studies that compared physical activity promotion with usual care or no intervention were considered for this review. The usual care intervention group received general information about the benefits of exercises and information about prediabetes and were not included in any form of the physical activity promotion programme.

\section{Outcome}

Studies were included when they measured the following outcome measures-adiponectin, leptin, CRP, IL-6 and TNF- $\alpha$.

\section{Types of studies}

In this review, we included randomized controlled trials.

\section{Data collection}

Data extraction form was arranged and pilot-tested to extract the study details. Parameters extracted from the included studies were author, year, journal, study design, sample size, age, gender, length of intervention, frequency and intensity of intervention, control and experimental group intervention description, details of the outcome measured and results (online resource 3). For continuous outcomes from randomized controlled trials, we recorded a sample size in each intervention group, mean and standard deviation (SD), mean and standard error (SE), mean and $95 \%$ confidence interval (CI) and median and interquartile range (IQR).

\section{Assessment of risk of bias in included studies}

Two authors independently assessed risk of bias of included studies, and the third author resolved disagreements. To assess the risk of bias, we used the Cochrane collaborations tool for risk of bias [23]. Risk of bias assessment was made at the study level. Each included study was assessed on the following key domains: random sequence generation, allocation concealment, blinding, selective outcome reporting, incomplete outcome data and any other risk of bias. Other sources of risk of bias assessment include deviation from study protocol, inappropriate intervention, insensitive instrument, baseline imbalance, contamination and if the study affected by interim results. Each domain was assessed at low, high or unclear risk of bias. The overall risk of bias of each study was assessed at low risk when all the key domains were assessed at low risk, high risk when one or more key domains were assessed at high risk and unclear risk when one or more key domains were assessed at unclear risk of bias.

\section{Data synthesis}

For continuous outcomes, the results were reported as mean difference (MD) with 95\% confidence interval (CI). For the studies reporting the median and interquartile range, the values converted to mean and standard deviations by using the appropriate method [24]. In studies with more than two intervention groups, the groups were combined to form a single pairwise comparison using the method set out in the Cochrane handbook [25].

We used Review Manager 5.3 software to perform a meta-analysis. Data available from trails for the outcomes of interest of this review were synthesized using the generic inverse variance method to derive the pooled estimates for continuous variables. Generic inverse variance method was preferred as the included studies reported 'adjusted' estimate of treatment effect. Random effects model was used in anticipation of substantial heterogeneity between included studies. The level of heterogeneity was determined by assessing $\mathrm{Tau}^{2}, \mathrm{I}^{2}$ and $\mathrm{Chi}^{2}$ statistics. Substantial heterogeneity was considered significant when $\mathrm{p}<0.05$ in $\mathrm{Chi}^{2}$ test and $\mathrm{I}^{2}>60 \%$ and $\mathrm{Tau}^{2}$ is greater than zero. Results with high heterogeneity were interpreted with caution. To explore the heterogeneity, subgroup analysis was not performed as the number of included studies was less than ten.

\section{Sensitivity analysis}

We performed a sensitivity analysis to explore the influence of nonparametric data on effect size by restricting the analysis using four studies reporting parametric data.

\section{Grade assessment}

Two authors have independently assessed the quality of evidence using the GRADE approach with GRADE PRO GDT software [26].

\section{Results}

\section{Search results}

Our search retrieved 1,688 citations that included electronic and manual search. One thousand two hundred and fortyfive $(1,245)$ records screened after duplicate removal. After filtering citations by title screening and abstract screening, 31 full-text articles were assessed for eligibility of inclusion. Nine studies satisfied the pre-specified criteria for inclusion and were included in the review, and eight studies have provided data for meta-analysis. One study was excluded from the meta-analysis as the sample originated from the same 
study, and the outcome (CRP) was published in two different reports [27]. Details are explained in Fig. 1

\section{Included studies}

\section{Study design}

For this review, we included nine studies. All included studies were randomized controlled trials (online resource 3).

\section{Participants}

One thousand nine hundred and six $(1,906)$ participants were included in this systematic review with the age group ranging from 20 to 70 years. All participants were diagnosed by either WHO or ADA.

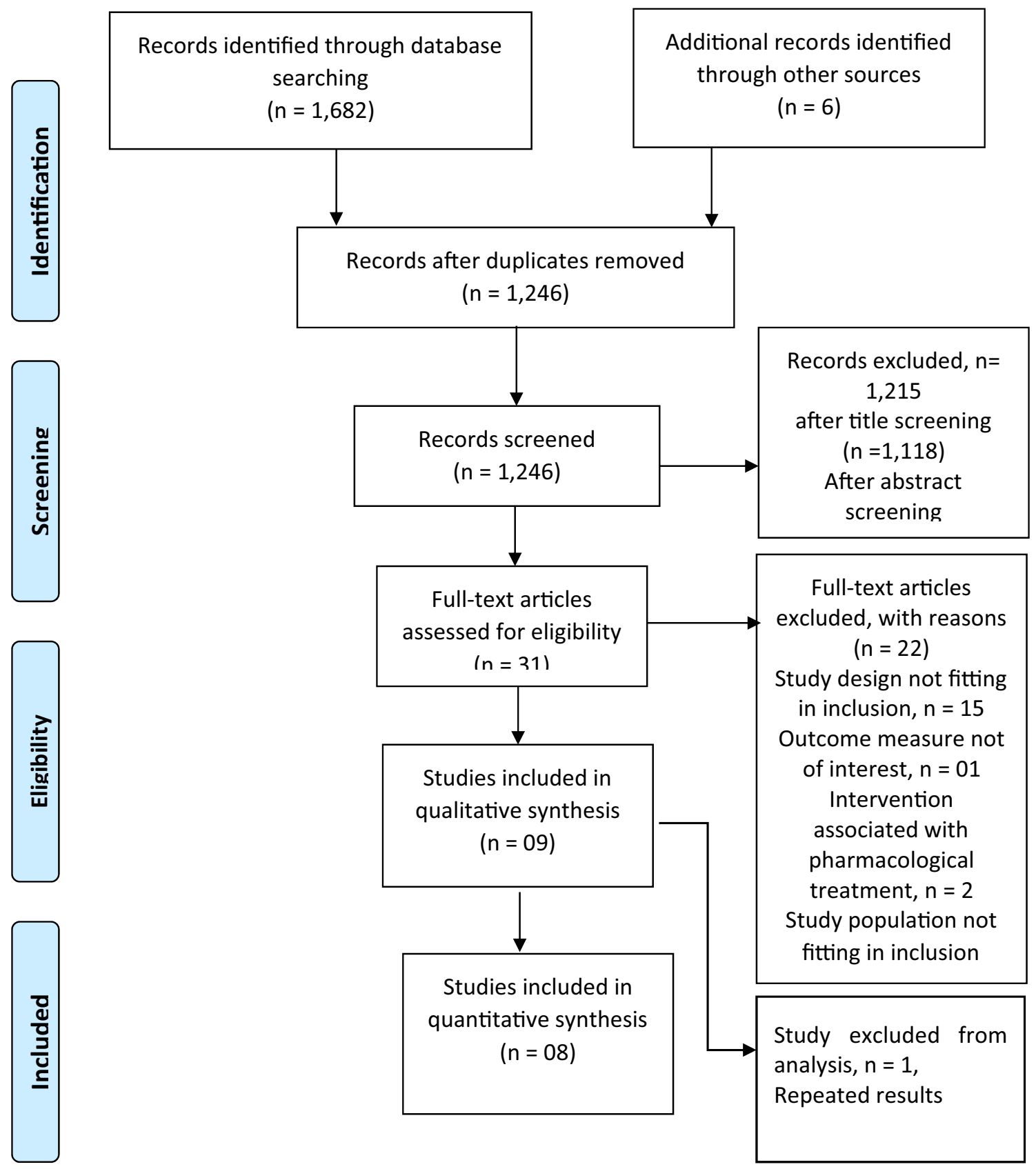

Fig. 1 PRISMA flow chart 


\section{Description of intervention}

Eight studies compared the effect of different physical activity (with or without dietary and lifestyle modifications) with usual care on different inflammatory markers in individuals with prediabetes. One study compared the effect of physical activity (with or without dietary and lifestyle modifications) with no intervention on inflammatory markers in individuals with prediabetes.

\section{At intervention}

Two studies encouraged physical activities along with diet as a part of the study intervention [20,28]. Two encouraged $140 \mathrm{~h}$ of scheduled activities [18, 27]. In one study, physical activity promotion was aimed to give a weight loss programme along with dietary modifications [19]. Other physical activity interventions consisted of moderate to vigorous exercises $\geq 30 \mathrm{~min} /$ day [29], walking plus resistance exercise [30], nordic walking [31] and physical activity promotion with and without pedometer [32]. The duration of the intervention varied between included studies. Different durations of intervention were 3 months, 6 months, 1 year and 5 years.

\section{At control}

Participants received advice on the benefits of exercises and general information about lifestyle, diabetes risk, and information about the consequences and symptoms of impaired glucose tolerance.

\section{Comparisons}

The included studies provided data for two comparisons.

\section{Comparison 1}

Physical activity with or without dietary or lifestyle modification versus usual care.

Eight studies provided data for this comparison.

\section{Comparison 2}

Physical activity with or without dietary or lifestyle modification versus no intervention.

Only one study provided data for this comparison.

\section{Outcome measures}

Total nine studies were included to measure the effect of an intervention on adiponectin, leptin, CRP, IL- 6 and TNF- $\alpha$. Five out of nine studies reported adiponectin as one of their outcome measures [19, 20, 28, 31, 32]. Effect of physical activity on leptin was studied in six studies [18-20, 28, 31, 32]. CRP was studied in five $[18,19,27,29,30]$ and IL-6 in five studies [19, 20, 29-31]. TNF- $\alpha$ was measured in three included studies [19, 20,31].

\section{Unit of measurement}

We noted that variations in reporting unit of measurement for adiponectin and CRP outcomes. Hence, we converted these values to a standard unit of measurement for each outcome before conducting the meta-analysis.

Adiponectin was reported in different units (ng/ml; ng/l and $\mu \mathrm{g} / \mathrm{ml}$ ) of measurement in the included studies. We converted the adiponectin values of the studies to $\mu \mathrm{g} / \mathrm{ml}[19$, 20, 32].

CRP values in included studies were reported in $\mathrm{mg} / \mathrm{l}[18$, $29,30]$. We converted the CRP values of one study from $\mathrm{mg}$ / $\mathrm{ml}$ to $\mathrm{mg} / \mathrm{l}[19]$.

\section{Combining groups}

One study reported leptin values separately for males and females [18]. These subgroup data were combined to form a single group for both intervention and control groups using the method suggested in the Cochrane handbook (Chapter 7, Sect. 7.7.a).

Three studies compared more than two intervention groups [30-32]. Two of the intervention groups from these studies were combined to form a single intervention group using the method suggested in the Cochrane handbook (Chapter 7, Sect. 7.7.a).

\section{Risk of bias of included studies}

Among the nine included studies in this review, two studies $[20,30]$ had a low overall risk of bias assessment. Four studies $[19,28,29,31]$ had a high overall risk of bias, and three studies [18, 27, 32] had an unclear overall risk of bias.

We assessed seven studies at low risk of selection bias [18-20, 28-31] as they adequately reported sequence generation for recruiting participants. Two studies [27, 32] were assessed at unclear risk of bias. Two studies $[20,30]$ adequately reported the method used for allocation of participants and were assessed at low risk of bias. Five studies [18, $19,27-29,32]$ were assessed at unclear risk and one study [31] was assessed at high risk as the study did not effectively conceal the allocation of participants to intervention groups.

Due to the type of intervention, blinding of participants and professionals providing treatment was not feasible; hence, this domain was assessed at low risk of bias. Similarly, we assessed blinding of outcome assessors at low risk of bias as the numerical values of the outcome of interest 
were obtained from independent laboratory tests and thus would not influence the intervention effect estimates.

For reporting bias, four studies [20, 27, 30, 31] had a published protocol and there was no indication of selective reporting and hence were assessed at low risk. Three studies $[18,28,32]$ were assessed at unclear risk, as there was no registered protocol available. We judged two studies [19, 29] at high risk for reporting outcomes of interest of this review that were not included as a part of the trial protocol.

For incomplete outcome data, we assessed two studies $[28,29]$ at high risk as the dropout rate was more than $20 \%$. We did not identify any other potential sources of bias from the included studies (online resource 4).

\section{Effects of intervention}

\section{Comparison 1: physical activity with or without dietary or lifestyle modification versus control}

Seven studies contributed to this comparison [18-20, 28-31].

\section{Adiponectin $(\mu \mathrm{g} / \mathrm{ml})$}

Four studies measured adiponectin levels after three months [31], four months [20] and one year [19, 28] of intervention. Physical activity did not have any effect on adiponectin levels (Fig. 2: Analysis 1.1) (mean difference (MD) $0.26 \mu \mathrm{g} /$ ml, $95 \%$ CI -0.42 to 0.93 ; participants $=398$; four studies, $\mathrm{I}^{2}=58 \%$, certainty of the evidence is very low).

The sensitivity analysis was conducted by excluding one study [28]. The results suggest the adiponectin levels may increase in the physical activity intervention group as compared to the usual care group (MD $0.69 \mu \mathrm{g} / \mathrm{ml}, 95 \%$ CI -0.62 to 2.00 ; participants $=295$; three studies, $\mathrm{I}^{2}=69 \%$ ).

\section{Leptin $(\mathrm{ng} / \mathrm{ml})$}

Five studies measured leptin levels after three months [31], four months [20], one year [19, 28] and one study after five years [18] of intervention. Physical activity reduced the leptin levels in prediabetic individuals (Fig. 3: Analysis 1.2) (MD $-2.11 \mathrm{ng} / \mathrm{ml}, 95 \% \mathrm{CI}-3.81$ to -0.42 ; participants $=566$; five studies, $\mathrm{I}=83 \%$, certainty of the evidence is very low).

The sensitivity analysis was conducted by excluding one study [28]. We observed that the direction of effect of intervention did not change for leptin (MD - $2.05 \mathrm{ng} / \mathrm{ml}, 95 \% \mathrm{CI}$ -4.11 to -0.01 ; participants $=463$; four studies, $\mathrm{I}^{2}=80 \%$ ).

\section{C-reactive protein (CRP) (mg/l)}

Four studies measured CRP levels after one year [19, 29, 30] and one study after five years [18] of intervention. Physical activity did not have any effect on the CRP levels in prediabetic individuals (Fig. 4: Analysis 1.3) (MD -0.05 mg/l,

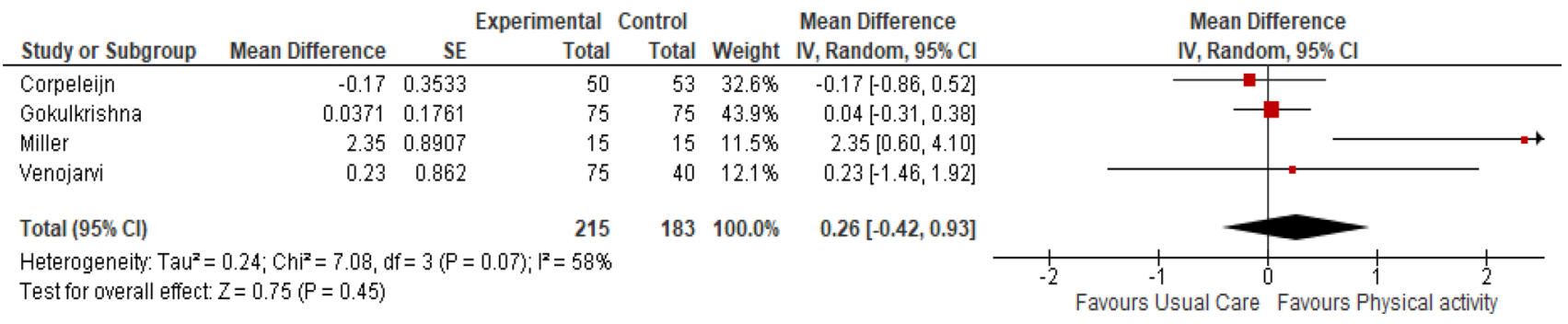

Fig. 2 Analysis 1.1: effect of physical activity with or without dietary or lifestyle modification versus usual care on adiponectin

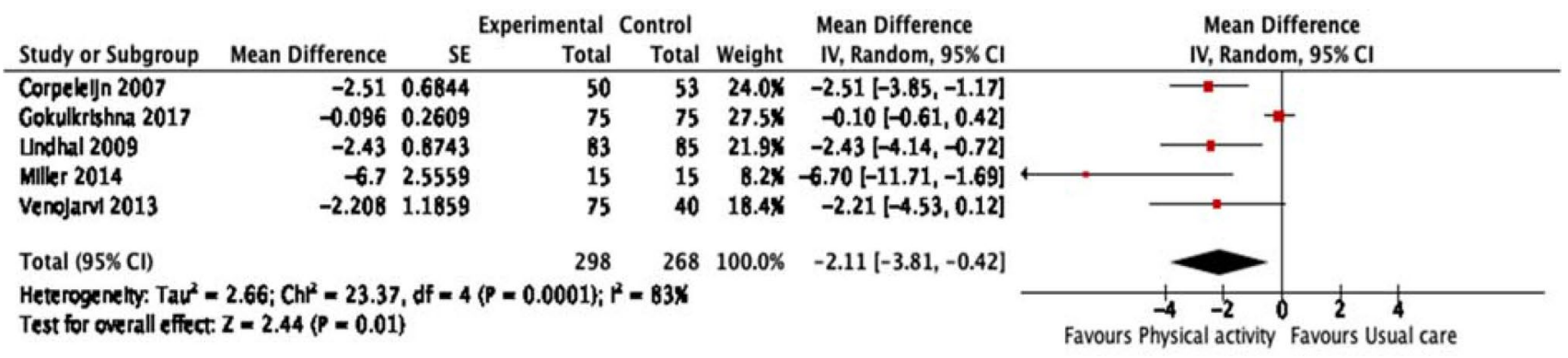

Fig. 3 Analysis 1.2: effect of physical activity with or without dietary or lifestyle modification versus usual care on leptin 
$95 \%$ CI -0.33 to 0.23 , four studies, 678 participants, $\mathrm{I}^{2}=0 \%$, certainty of the evidence is low).

The sensitivity analysis was conducted by excluding two studies [29, 30]. We observed that the direction of effect of intervention did not change for CRP (MD $-0.25 \mathrm{mg} / 1,95 \%$ CI -0.78 to 0.28 , two studies, 198 participants, $\mathrm{I}^{2}=0 \%$ ).

\section{Interleukin- 6 (IL-6) (pg/ml)}

Five studies measured IL-6 after one year [19, 29, 30], four months [20] and one study after three months [31] of intervention. Physical activity reduced the interleukin-6 levels in prediabetic individuals (Fig. 5: Analysis 1.4) (MD -0.15 pg/ $\mathrm{ml}, 95 \% \mathrm{CI}-0.25$ to -0.04 ; participants $=775$; five studies, $\mathrm{I}^{2}=0 \%$, certainty of the evidence is low).

The sensitivity analysis was conducted by excluding two studies $[29,30]$. We observed that the direction of effect of intervention did not change for IL-6 (MD - $0.20 \mathrm{pg} / \mathrm{ml}, 95 \%$ CI -0.33 to -0.07 ; participants $=295$; three studies, $\mathrm{I}^{2}=0 \%$ ).

\section{Tumour necrosis factor- $a$ (TNF-a) (pg/ml)}

Three studies measured TNF- $\alpha$ after three months [31], four months [20] and one year [19] of intervention. Physical activity did not have any effect on TNF- $\alpha$ in prediabetic individuals (Fig. 6: Analysis 1.5) (MD 0.67 pg/ml, 95\% CI -2.56 to 3.89; participants $=295$; three studies, $\mathrm{I}^{2}=69 \%$, certainty of the evidence is very low).

\section{Comparison 2: physical activity with or without dietary or lifestyle modification versus no intervention}

Only one study provided data for this comparison and provided two outcomes: adiponectin and leptin [32].

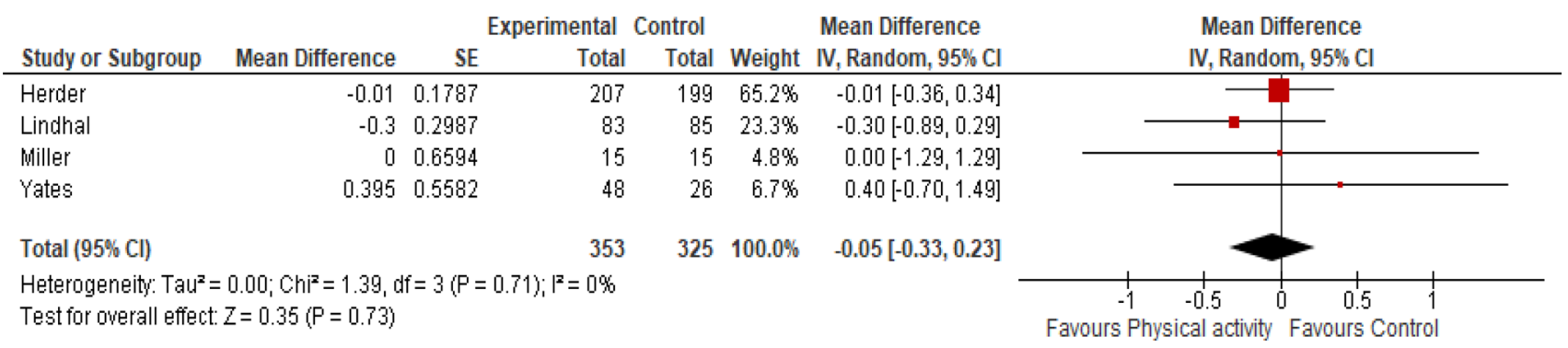

Fig. 4 Analysis 1.3: effect of physical activity with or without dietary or lifestyle modification versus usual care on CRP

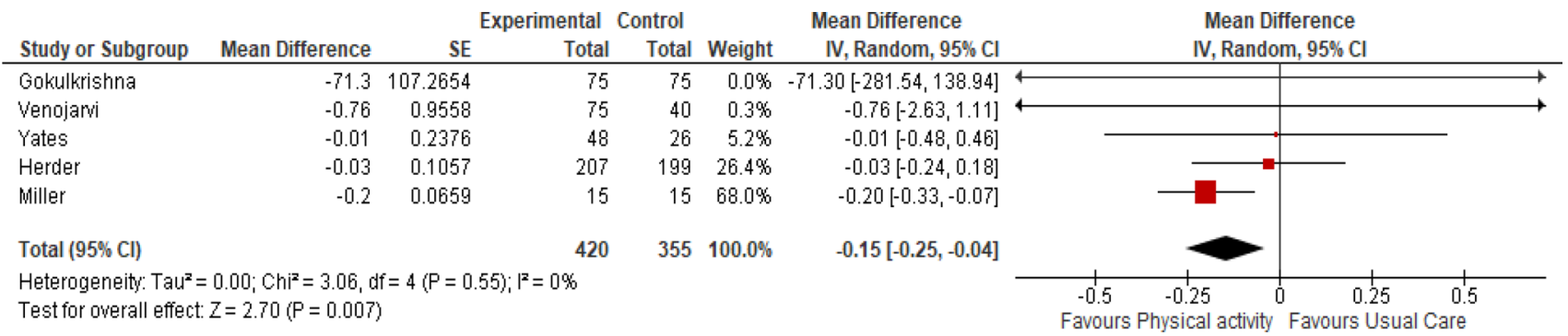

Fig. 5 Analysis 1.4: effect of physical activity with or without dietary or lifestyle modification versus usual care on IL-6

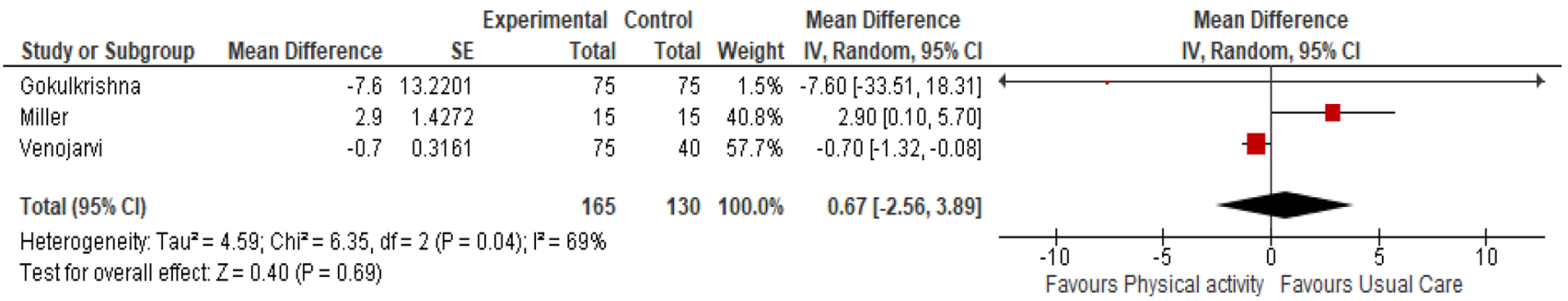

Fig. 6 Analysis 1.5: effect of physical activity with or without dietary or lifestyle modification versus usual care on TNF- $\alpha$ 


\section{Adiponectin $(\mu \mathrm{g} / \mathrm{ml})$}

Physical activity intervention did not have any effect on adiponectin levels in prediabetic individuals (mean difference (MD) $1.30 \mu \mathrm{g} / \mathrm{ml}, 95 \% \mathrm{CI}-0.57$ to 3.17 , one study, 61 participants, certainty of the evidence is very low).

\section{Leptin (ng/ml)}

Physical activity intervention reduced the leptin levels in prediabetic individuals (MD $-2.08 \mathrm{ng} / \mathrm{ml}, 95 \% \mathrm{CI}-3.87$ to 0.29 , one study, 61 participants, certainty of the evidence is very low).

\section{Discussion}

In this review, we investigated the effect of physical activity promotion programme on inflammatory markers in individuals with prediabetes. We included nine trials with total 1,906 participants. The meta-analysis was performed to compare the effect of physical activity with or without dietary or lifestyle modification versus usual care (comparison 1) and physical activity with or without dietary or lifestyle modification versus no intervention (comparison 2) on inflammatory markers adiponectin, leptin, CRP, IL- 6 and TNF- $\alpha$. Out of the nine studies, we included eight studies for metaanalysis; seven studies were included in the first comparison, and one study was included in the second comparison. In the first comparison, the physical activity intervention administered along with or without dietary or lifestyle modification may reduce the level of leptin and IL-6 in individuals with prediabetes. On the other hand, we are uncertain if the intervention would affect the level of adiponectin, CRP and TNF$\alpha$. In the second comparison, only one study provided data and we found that physical activity with or without dietary or lifestyle modification, when compared to the group that did not receive any form of intervention, had no effect on the level of adiponectin, but it reduces the level of leptin in individuals with prediabetes.

The grade assessment for certainty of evidence was very low for adiponectin, CRP and TNF- $\alpha$ (no effect); very low for leptin and low for IL-6 (favours physical activity) in the first comparison. The studies were downgraded for certainty of evidence mainly due to inconsistency, imprecision, risk of bias. For the second comparison, only one study reported adiponectin and leptin outcomes (summary of findings presented in online resource 5).

As per Cochrane risk of bias tool, we assessed trials as low, high or unclear risk. We contacted all the authors of the included trial to confirm our judgement. Nearly $25 \%$ of the studies had insufficient information on sequence generation, and about half of the studies had no information on methods used to conceal allocation. Less than $15 \%$ of the studies were assessed at high risk for inadequate allocation concealment. High attrition rates were reported in less than $25 \%$ of the studies. Majority of the included studies had inadequate reporting of outcomes of interest.

We have identified previously published systematic reviews and meta-analysis that evaluated the effect of physical activity on various inflammatory markers. Becic et al. 2018 included individuals with diabetes and prediabetes and found that physical exercise reduced leptin levels and increased adiponectin levels [33]. Yu N et al. 2016 evaluated the effect of exercise on leptin and adiponectin in overweight and obese individuals. They also found that exercise reduced leptin levels and increased adiponectin levels [34]. Serico et al. 2018 investigated that physical exercise without any concomitant dietary intervention improved leptin, adiponectin and IL-6 in children with obesity [35]. Anche et al. 2020 investigated the effect of lifestyle modification and physical activity promotion on leptin in individuals with metabolic syndrome included both randomized and non-randomized trials [36]. Zeng et al. 2019 assessed the effect of aerobic exercises on inflammatory markers in healthy middle-aged and elderly adults suggested a positive effect of an intervention on CRP, TNF- $\alpha$ and IL- 6 [37]. Our review differed from the previous review in terms of population and the interventions. We included only prediabetes population as per ADA or WHO criteria between the age group of 20 to 70 years, and the intervention focused mainly on the physical activity promotion programme administered along with or without dietary or lifestyle modifications. Hence, the evidence of this review does not apply to the paediatric population or normoglycaemic adults.

Effect of exercise on pro-inflammatory cytokines has been inconsistent. The different reasons include health status, age, sex, disease, type of exercise, duration and intensity of exercise [38]. Changes in the level of inflammatory markers due to physical activity promotion are directly proportional to the mode, duration and intensity of an activity. Combined exercise has a better anti-inflammatory effect than aerobic or resistance exercise alone [39]. There was dissimilarity in the type of interventions in terms of its mode, duration, type of monitoring and intensity administered in the studies included in this review. This could be one of the reasons for finding the different results on the included outcome measures. However, it should also be noted that there were very few studies that contributed data to each outcome and the included studies had small sample size which could have shown a greater effect of the intervention and were possibly underpowered to detect the desired effect of the intervention on multiple outcomes. Sensitivity analysis of adiponectin, CRP and TNF- $\alpha$ suggests that the intervention did have a positive effect on adiponectin levels, while the other outcomes did not show any change. Due to the limited number of studies, we could not perform 
a sensitivity analysis by excluding studies with a high risk of bias and subgroup analysis to explore the heterogeneity.

We found that physical activity promotion with or without dietary or lifestyle modification did not affect adiponectin level, however showed a positive effect on leptin in individuals with prediabetes. There exists a dose-response relationship of adiponectin and leptin with weight loss in human participants indicating that mild weight loss up to $5 \%$ reduces the leptin level but to make a change in the level of adiponectin weight reduction should be more than $10 \%$ [40]. Prediabetes stage is associated with the increase in the level of inflammatory markers, that is, CRP, IL-6 and TNF- $\alpha[9,10,13,41]$. IL- 6 promotes the expression of leptin on mRNA and inhibits the expression of adiponectin. This explains the impact of physical activity on adiponectin and leptin by reducing IL-6 [42]. Weight reduction causes a reduction in the size of adipocyte and reduction in the secretions of inflammatory cytokines. The concentration of IL-6 correlates with the size of adipocyte [43]. However, secretion of TNF- $\alpha$ is independent of the size of adipocyte [44, 45]. The literature on cytokine production has also proposed that IL-6 suppresses the production of TNF- $\alpha$ [46].

\section{Limitations}

This review has few limitations; we only considered the published literature and English language. Grey literature or research registry was not incorporated into the search. However, we could not assess publication bias using a funnel plot, as we had less than ten studies qualified for inclusion. There are very few studies included in this review under each outcome and comparison to provide recommendations for clinical applicability.

\section{Implication for future research}

Physical activity promotion may reduce the inflammation in the individuals with prediabetes; however, level of its evidence is very low; also there is a dearth of literature on minimally clinical important difference (MCID) value for considered outcome measures. Further trials are needed to focus on this specific question with robust sample size; methodological rigour and long-term follow-up to estimate the effect of physical activity promotion on inflammatory markers in individuals with prediabetes.

\section{Conclusion}

This review suggests that physical activity promotion programme may reduce the level of leptin and IL-6, but it is uncertain whether there is any effect on the level of adiponectin, CRP and TNF- $\alpha$ in individuals with prediabetes.
Acknowledgement We would like to acknowledge Centre for Diabetic Foot Care and Research for a support.

Author contributions Idea of article was provided by GAM and RAJ. $\mathrm{SU}, \mathrm{KNS}$ and RAJ performed a literature search. AH performed the analysis, interpretation of result, RAJ and AH independently performed ROB assessment and Grade assessment. RAJ, AH, GAM drafted the manuscript. GAM, SU, KNS critically revised the manuscript.

Funding Open access funding provided by Manipal Academy of Higher Education, Manipal. This study is not funded by any research grant or any funding agency.

Data availability All the data generated and analysed during this review have been provided in article and in supplementary files.

Code availability Not applicable.

\section{Compliance with ethical standards}

Conflict of interest Ms. Radhika Aditya Jadhav, Dr. Arun G Maiya, Ms. Aditi Hombali, Dr. Shashikiran Umakanth and Dr. Shivashankar $\mathrm{K} \mathrm{N}$ declared that they do not have any conflict of interest.

Ethical approval Not applicable.

Informed consent Not applicable.

Open Access This article is licensed under a Creative Commons Attribution 4.0 International License, which permits use, sharing, adaptation, distribution and reproduction in any medium or format, as long as you give appropriate credit to the original author(s) and the source, provide a link to the Creative Commons licence, and indicate if changes were made. The images or other third party material in this article are included in the article's Creative Commons licence, unless indicated otherwise in a credit line to the material. If material is not included in the article's Creative Commons licence and your intended use is not permitted by statutory regulation or exceeds the permitted use, you will need to obtain permission directly from the copyright holder. To view a copy of this licence, visit http://creativecommons.org/licenses/by/4.0/.

\section{References}

1. Tabák AG, Herder C, Rathmann W et al (2012) Prediabetes: a high-risk state for diabetes development. Lancet 379:2279-2290. https://doi.org/10.1016/S0140-6736(12)60283-9

2. ADA - American Diabetes Association (2012) Diagnosis of diabetes and prediabetes. Diabetes Care 35:11-63

3. World Health Organization (2006) Definition and diagnosis of diabetes mellitus and intermediate hyperglycemia. Report of a WHO Study, Diabetes Mellitus

4. Buysschaert M, Bergman M (2011) Definition of prediabetes. Med Clin North Am 95:289-297

5. Hamdy O1, Porramatikul-OE SA (2012) Metabolic obesity: the paradox between visceral and subcutaneous fat. Curr Diabetes Rev 2:367-373. https://doi.org/10.2174/1573399810602040367

6. Haffner SM (2003) Pre-diabetes, insulin resistance, inflammation and CVD risk. Diabetes Res Clin Pract 61:S9-S18. https://doi. org/10.1016/S0168-8227(03)00122-0 
7. Berg AH, Scherer PE (2005) Adipose tissue, inflammation, and cardiovascular disease. Circ Res 96:939-949

8. Halberg N, Wernstedt-Asterholm I, Scherer PE (2008) The adipocyte as an endocrine cell. Endocrinol Metab Clin North Am 37:753-768

9. Huang Z, Chen C, Li S et al (2016) Serum markers of endothelial dysfunction and inflammation increase in hypertension with prediabetes mellitus. Genet Test Mol Biomarkers 20:322-327. https ://doi.org/10.1089/gtmb.2015.0255

10. Jiang Y, Owei I, Wan J et al (2016) Adiponectin levels predict prediabetes risk: the pathobiology of prediabetes in a biracial cohort (POP-ABC) study. BMJ Open Diabetes Res Care 4:18-22. https ://doi.org/10.1136/bmjdrc-2016-000194

11. Ghantous CM, Azrak Z, Hanache S et al (2015) (2015) Differential role of leptin and adiponectin in cardiovascular system. Int. J. Endocrinol 6:1-13

12. Kwon H, Pessin JE (2013) Adipokines mediate inflammation and insulin resistance. Front Endocrinol (Lausanne) 4:71

13. Huang Y, Cai X, Mai W et al (2016) Association between prediabetes and risk of cardiovascular disease and all cause mortality: systematic review and meta-analysis. BMJ. https://doi. org/10.1136/bmj.i5953

14. Cloostermans L, Wendel-Vos W, Doornbos G et al (2015) Independent and combined effects of physical activity and body mass index on the development of type 2 diabetes - a metaanalysis of 9 prospective cohort studies. Int J Behav Nutr Phys Act. https://doi.org/10.1186/s12966-015-0304-3

15. Swindell N, Rees P, Fogelholm M et al (2020) Compositional analysis of the associations between $24 \mathrm{~h}$ movement behaviours and cardio-metabolic risk factors in overweight and obese adults with pre-diabetes from the PREVIEW study: cross-sectional baseline analysis. Int J Behav Nutr Phys Act 17:1-12. https:// doi.org/10.1186/s12966-020-00936-5

16. Ramachandran A, Snehalatha $C$, Mary S et al (2006) The Indian diabetes prevention programme shows that lifestyle modification and metformin prevent type 2 diabetes in Asian Indian subjects with impaired glucose tolerance (IDPP-1). Diabetologia 49:289-297. https://doi.org/10.1007/s00125-005-0097-z

17. Jadhav RA, Hazari A, Monterio A et al (2017) Effect of physical activity intervention in prediabetes: a systematic review with meta-analysis. J Phys Act Heal 14:745-755

18. Lindahl B, Nilsson TK, Borch-Johnsen K et al (2009) A randomized lifestyle intervention with 5-year follow-up in subjects with impaired glucose tolerance: pronounced short-term impact but long-term adherence problems. Scand J Public Health $37: 434-442$

19. Miller GD, Isom S, Morgan TM et al (2015) Effects of a community-based weight loss intervention on adipose tissue circulating factors. Diabetes Metab Syndr 8:205-211. https://doi. org/10.1016/j.dsx.2014.09.003.Effects

20. Gokulakrishnan K, Ranjani H, Weber MB et al (2017) Effect of lifestyle improvement program on the biomarkers of adiposity, inflammation and gut hormones in overweight/obese Asian Indians with prediabetes. Acta Diabetol 54:843-852. https://doi. org/10.1007/s00592-017-1015-9

21. Moher D, Liberati A, Tetzlaff J et al (2009) Preferred reporting items for systematic reviews and meta-analyses: the PRISMA statement. PLoS Med. https://doi.org/10.1371/journ al.pmed. 1000097

22. Ouzzani M, Hammady H, Fedorowicz Z et al (2016) Rayyan-a web and mobile app for systematic reviews. Syst Rev. https:// doi.org/10.1186/s13643-016-0384-4

23. Higgins JPT, Thomas J, Chandler J, Cumpston M, et al Page MJ WV (editors) (2019) Chapter 8: Assessing risk of bias in a randomized trial. In: Cochrane Handbook for Systematic Reviews of
Interventions version 6.0 (updated July 2019). Cochrane, www. training.cochrane.org/handbook.

24. Wan X, Wang W, Liu J et al (2014) Estimating the sample mean and standard deviation from the sample size, median, range and/ or interquartile range. BMC Med Res Methodol. https://doi. org/10.1186/1471-2288-14-135

25. Higgins JPT DJ (editors) (2011) Chapter 7: Selecting studies and collecting data. In: Higgins JPT, Green S (editors). In: Cochrane Handbook for Systematic Reviews of Interventions Version 5.1.0 (updated March 2011). The Cochrane Collaboration. p www.handbook.cochrane.org.

26. GRADEpro Guideline Development Tool [Software]. McMaster University (2015) GRADEpro GDT. gradepro.org.

27. Andersson J, Boman K, Jansson JH et al (2008) Effect of intensive lifestyle intervention on $\mathrm{C}$-reactive protein in subjects with impaired glucose tolerance and obesity. Results from a randomized controlled trial with 5-years follow-up. Biomarkers 13:671-679. https://doi.org/10.1080/13547500802661266

28. Corpeleijn E, Feskens EJM, Jansen EHJM et al (2007) Lifestyle intervention and adipokine levels in subjects at high risk for type 2 diabetes: the study on lifestyle intervention and impaired glucose tolerance Maastricht (SLIM). Diabetes Care 30:3125-3127. https ://doi.org/10.2337/dc07-0457

29. Herder C, Peltonen M, Koenig W et al (2009) Anti-inflammatory effect of lifestyle changes in the Finnish Diabetes Prevention Study. Diabetologia 52:433-442. https://doi.org/10.1007/s0012 5-008-1243-1

30. Yates T, Davies MJ, Gorely T et al (2010) The effect of increased ambulatory activity on markers of chronic low-grade inflammation: evidence from the PREPARE programme randomized controlled trial. Diabet Med 27:1256-1263

31. Venojärvi M, Wasenius N, Manderoos S et al (2013) Nordic walking decreased circulating chemerin and leptin concentrations in middle-aged men with impaired glucose regulation. Ann Med 45:162-170. https://doi.org/10.3109/07853890.2012.727020

32. Liu Y, Zhang Z, Li J et al (2017) Changes of leptin, resistin, adiponectin and free fatty acid in the impaired glucose tolerance patients with exercise intervention. Biomed Res 28:6241-6247

33. Becic T, Studenik C, Hoffmann G (2018) Exercise increases adiponectin and reduces leptin levels in prediabetic and diabetic individuals: systematic review and meta-analysis of randomized controlled trials. Med Sci 6:97. https://doi.org/10.3390/medsc i6040097

34. Yu N, Ruan Y, Gao X et al (2017) Systematic review and meta-analysis of randomized, controlled trials on the effect of exercise on serum leptin and adiponectin in overweight and obese individuals. Horm Metab Res 49:164-173. https://doi. org/10.1055/s-0042-121605

35. Sirico F, Bianco A, D'Alicandro G et al (2018) Effects of physical exercise on adiponectin, leptin, and inflammatory markers in childhood obesity: systematic review and meta-analysis. Child Obes 14:207-217. https://doi.org/10.1089/chi.2017.0269

36. Anche P, Maiya AG, Kamath SU et al (2020) Lifestyle modification with physical activity promotion on leptin resistance and quality of life in metabolic syndrome - a systematic review with meta-analysis. Curr Diabetes Rev. https://doi.org/10.2174/15733 99816666200211102917

37. Zheng G, Qiu P, Xia R et al (2019) Effect of aerobic exercise on inflammatory markers in healthy middle-aged and older adults: a systematic review and meta-analysis of randomized controlled trials. Front Aging Neurosci. https://doi.org/10.3389/fnagi .2019 .00098

38. Taherkhani S, Suzuki K, Castell L (2020) A short overview of changes in inflammatory cytokines and oxidative stress in response to physical activity and antioxidant supplementation. Antioxidants 9:1-18. https://doi.org/10.3390/antiox9090886 
39. Hopps E, Canino B, Caimi G (2011) Effects of exercise on inflammation markers in type 2 diabetic subjects. Acta Diabetol 48:183189. https://doi.org/10.1007/s00592-011-0278-9

40. Klempel MC, Varady KA (2011) Reliability of leptin, but not adiponectin, as a biomarker for diet-induced weight loss in humans. Nutr Rev 69:145-154. https://doi.org/10.111 1/j.1753-4887.2011.00373.x

41. Van De Voorde J, Pauwels B, Boydens C et al (2013) Adipocytokines in relation to cardiovascular disease. Metabolism 62:1513-1521

42. Brandt C, Jakobsen AH, Adser H et al (2012) IL-6 regulates exercise and training-induced adaptations in subcutaneous adipose tissue in mice. Acta Physiol 205:224-235. https://doi.org/10.111 $1 / \mathrm{j} .1748-1716.2011 .02373 . x$

43. Mlinar B, Marc J (2011) New insights into adipose tissue dysfunction in insulin resistance. Clin Chem Lab Med 49:1925-1935

44. Hauner H, Bender M, Haastert B et al (1998) Plasma concentrations of soluble TNF-alpha receptors in obese subjects. Int J Obes 22:1239-1243. https://doi.org/10.1038/sj.ijo.0800773
45. Mohamed-Ali V, Goodrick S, Rawesh A et al (1997) Subcutaneous adipose tissue releases interleukin-6, but not tumor necrosis factor- $\alpha$, in vivo. J Clin Endocrinol Metab 82:4196-4200. https:// doi.org/10.1210/jcem.82.12.4450

46. Schindler R, Mancilla J, Endres S et al (1990) Correlations and interactions in the production of interleukin-6 (IL- 6), IL-1, and tumor necrosis factor (TNF) in human blood mononuclear cells: IL-6 suppresses IL-1 and TNF. Blood 75:40-47. https://doi. org/10.1182/blood.v75.1.40.40

Publisher's Note Springer Nature remains neutral with regard to jurisdictional claims in published maps and institutional affiliations. 